Supporting information

Real-Time Monitoring of Formation and Dynamics of Intra- and Interchain Phases in Single Molecules of Polyfluorene

Tzu-Wei Tseng ${ }^{1}$, Hao Yan $^{2}$, Tomonori Nakamura ${ }^{1}$, Shun Omagari ${ }^{1}$, Ji-Seon Kim ${ }^{2 *}$, Martin Vacha $^{1 *}$

${ }^{1}$ Department of Materials Science and Engineering, Tokyo Institute of Technology, Ookayama 2-12-1-S8-44, Meguro-ku, Tokyo 152-8552, Japan

${ }^{2}$ Department of Physics and Centre for Plastic Electronics, Imperial College London, South Kensington Campus, London SW7 2AZ, United Kingdom 

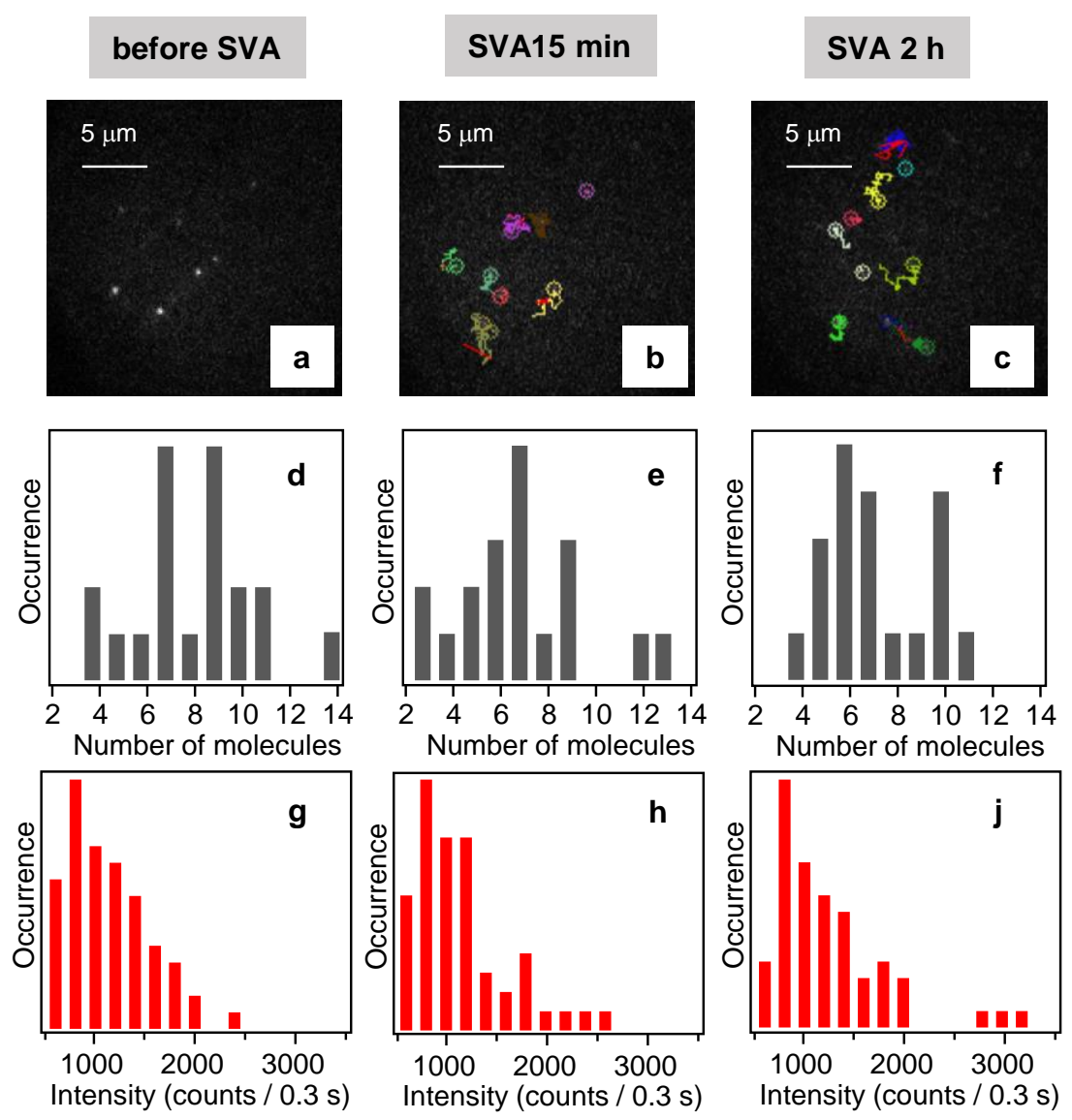

Figure S1. a) - c) Fluorescence images of low $\mathrm{M}_{\mathrm{w}}$ PFO dispersed in a PS thin film before (a) and under SVA with toluene for $15 \mathrm{~min}$ (b) and 2 hours (c). The color lines in b) and c) represent traces of the diffusing molecules; d) - f) Numbers of emitting spots (PFO chains) found in fluorescence images from 20 individual locations inside the samples shown in a) - c); g) - j) Fluorescence intensity distributions of single PFO chains analyzed from the 20 individual locations inside the samples shown in a) - c). 


\section{Analysis of the fluorescence images}

The images in Fig. S1a-c show a distribution of intensities in the from of bright and dark fluorescence spots. The fluorescence spectra from both the bright and darker spots are consistent with PFO spectra and prove that the fluorescence originates from PFO. The differences in the brightness can be explained by the large molecular weight distribution. From the values of $\mathrm{M}_{\mathrm{w}}$ and PDI it is possible to estimate the relative width $\sigma_{\mathrm{n}} / \mathrm{M}_{\mathrm{n}}=1.56$, where $\sigma_{\mathrm{n}}$ is a standard deviation. This ratio is much larger than the similar ratio $\sigma_{\mathrm{I}} /\left\langle I_{F}\right\rangle$ obtained from the distribution of fluorescence intensity in, e.g., Fig. S1g, which is on the order of $0.3 \sim 0.4$. Thus, the molecular weight distribution is much larger than the corresponding fluorescence intensity distribution. Further, the intensity shows a single-component distribution pointing to only the molecular weight distribution as the factor for the varying brightness. 

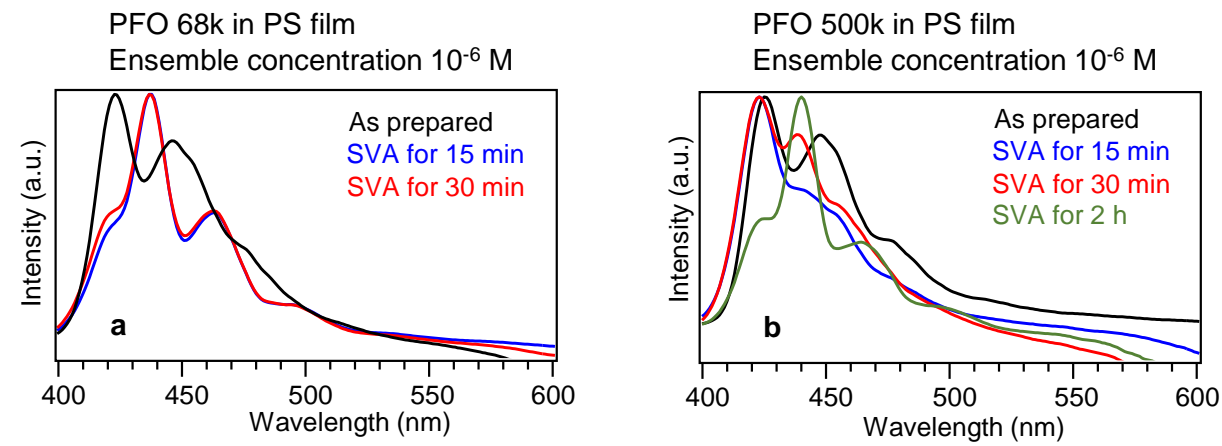

Figure S2. a) Fluorescence spectra of drop-cast films of PS doped with ensemble-level concentrations (on the order of $10^{-6} \mathrm{M}$ ) of low $\mathrm{M}_{\mathrm{w}}$ PFO before SVA and during SVA for different time periods. The SVA times are shown in the legend and the lines are distinguished by the corresponding colors. b) Same as a) for the high $\mathrm{M}_{\mathrm{w}}$ PFO. 


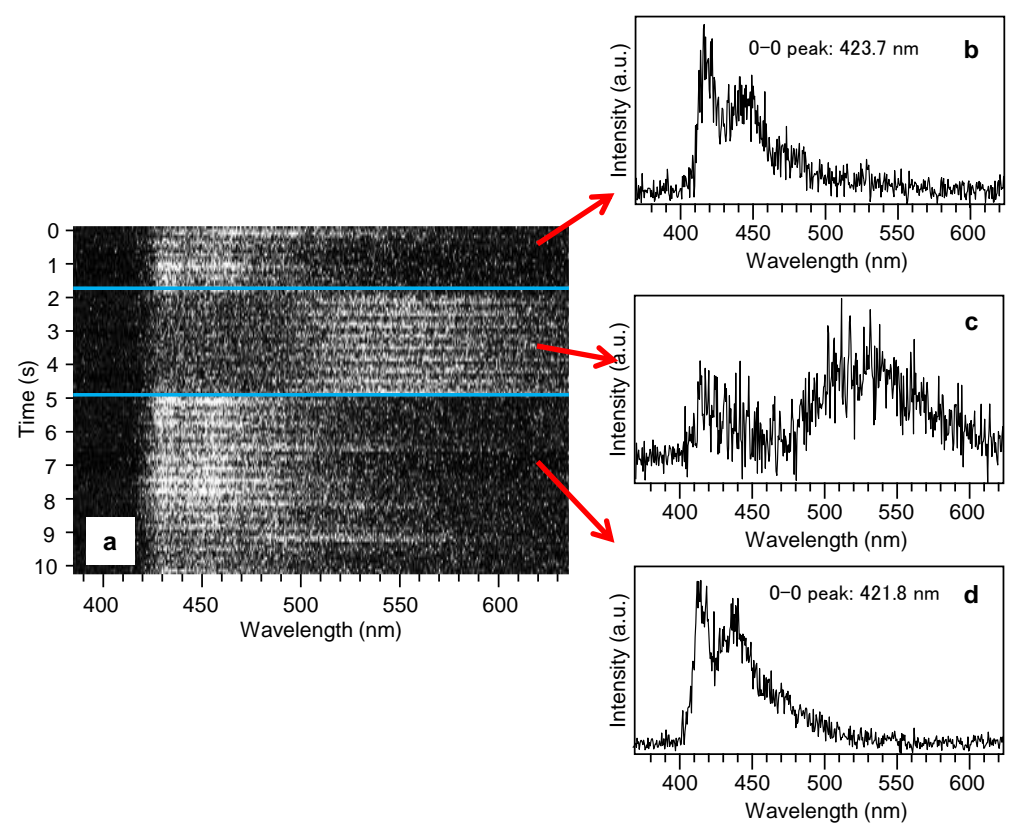

Figure S3. a) 2D fluorescence spectral plot of time evolution of a single low $\mathrm{M}_{\mathrm{w}} \mathrm{PFO}$ chain spectra, showing spontaneous switching between the glassy phase and the aggregation-induced green emission band; b) - d) Stationary spectra from the same PFO chain obtained from the time intervals indicated by the arrows in a). 


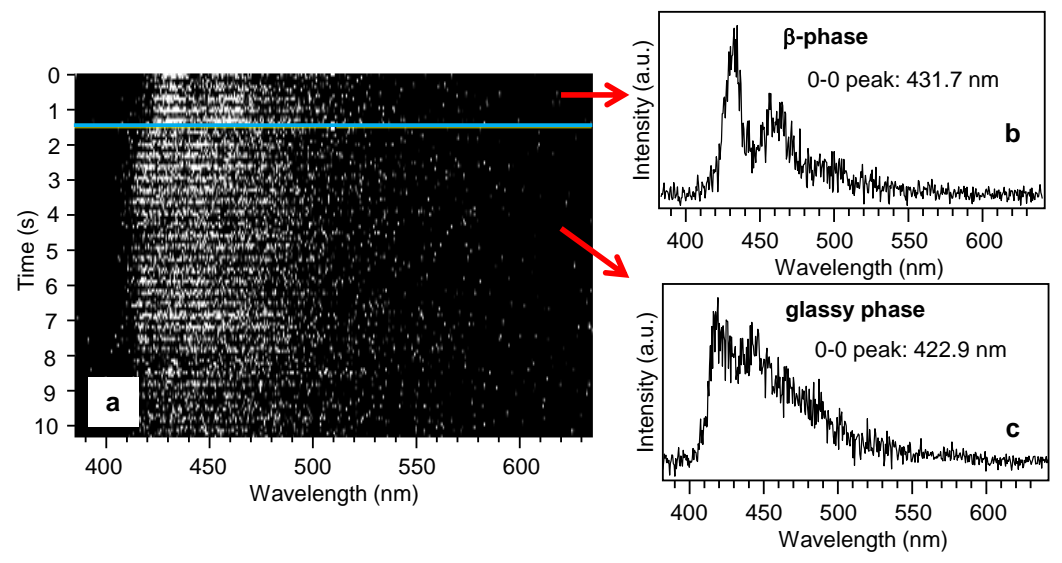

Figure S4. a) 2D fluorescence spectral plot of time evolution of a single low $\mathrm{M}_{\mathrm{w}} \mathrm{PFO}$ chain, showing spontaneous switching from $\beta$-phase to glassy phase emission; b), c) Stationary spectra from the same PFO chain obtained from the time intervals indicated by the arrows in a). 

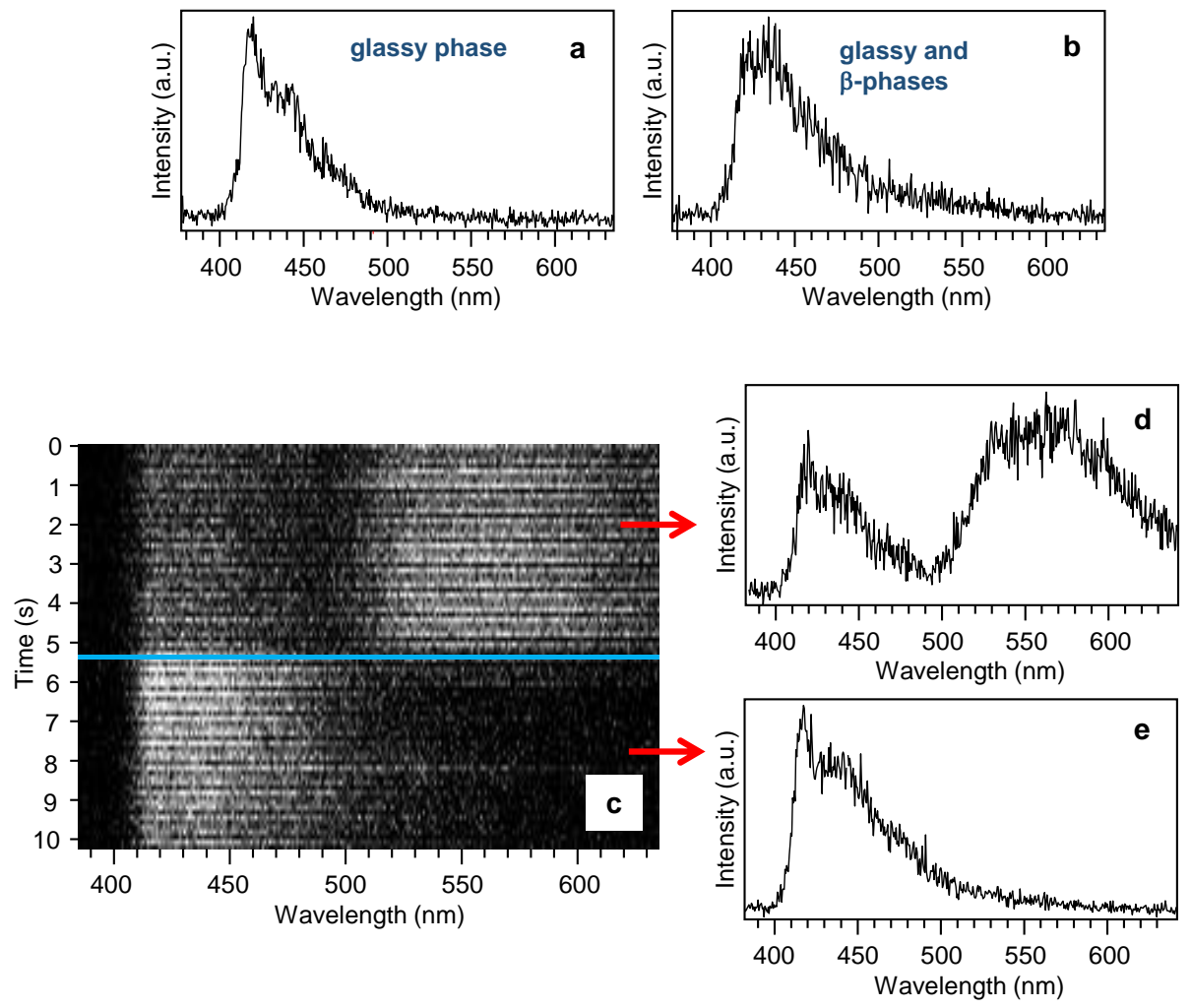

Figure S5. a), b) Fluorescence spectra from single high $\mathrm{M}_{\mathrm{w}}$ PFO chains after 15 min SVA, showing the glassy phase (a) or mixed glassy and $\beta$-phase character (b); c) 2D fluorescence spectral plot of time evolution of a single high $\mathrm{M}_{\mathrm{w}}$ PFO chain spectra, showing spontaneous switching between the glassy phase and the aggregation-induced green emission band; d), e) Stationary spectra from the same PFO chain obtained from the time intervals indicated by the arrows in c). 

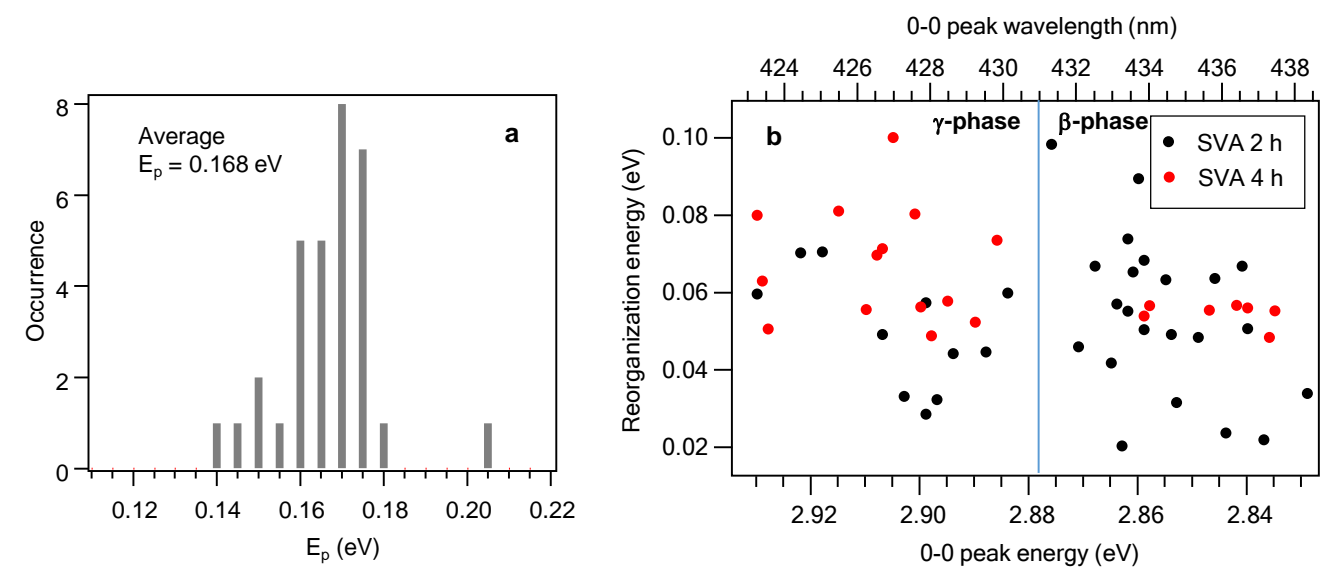

Figure S6. a) Distribution of the phonon energies of the 0-1 spectral line obtained from the fitting procedure as a difference between the $0-0$ and $0-1$ peaks; the energies correspond approximately to several Raman modes in the $1100 \mathrm{~cm}^{-1}-1400 \mathrm{~cm}^{-1}$ range; b) Reorganization energy $S E_{\mathrm{p}}$ plotted against the 0-0 peak energy. Black symbols - SVA for 2 hours, red symbols - SVA for 4 hours. 


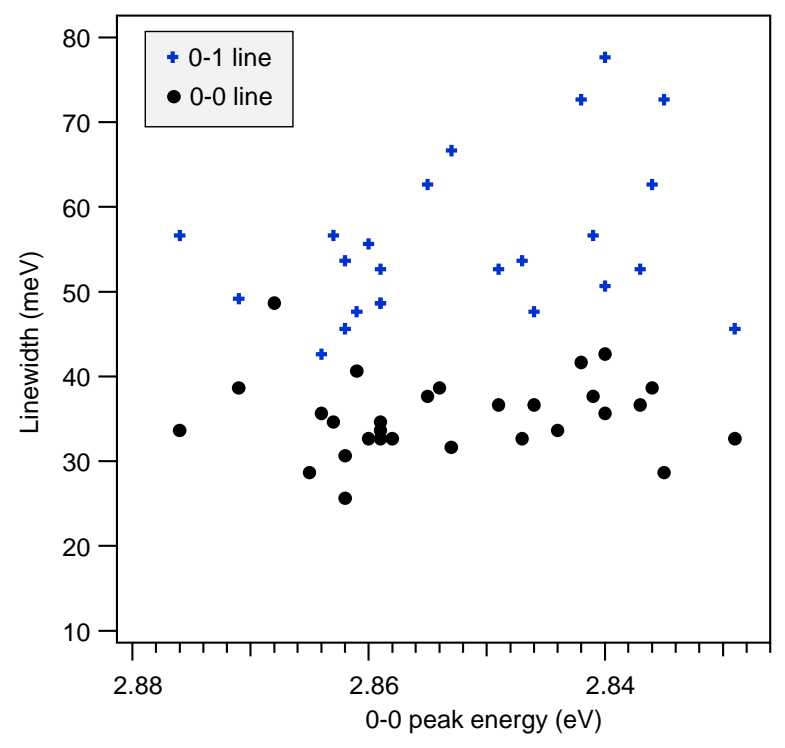

Figure S7. Linewidths of the $0-0$ and $0-1$ fluorescence peaks plotted versus the $0-0$ peak energy for the high $\mathrm{M}_{\mathrm{w}}$ PFO single chains in the $\beta$-phase spectral region. 